\title{
Études/Inuit/Studies
}

WEINSTEIN, Charles Arctique Extrême. Les Tchouktches du détroit de Béring, Éditions Autrement, Collection Monde n 117.

\section{Cesare Censi}

Volume 26, numéro 2, 2002

Populations et migrations

Populations and Migrations

URI : https://id.erudit.org/iderudit/007659ar

DOI : https://doi.org/10.7202/007659ar

Aller au sommaire du numéro

\section{Éditeur(s)}

Association Inuksiutiit Katimajiit Inc.

ISSN

0701-1008 (imprimé)

1708-5268 (numérique)

Découvrir la revue

Citer ce compte rendu

Censi, C. (2002). Compte rendu de [WEINSTEIN, Charles Arctique Extrême. Les Tchouktches du détroit de Béring, Éditions Autrement, Collection Monde

n 117.] Études/Inuit/Studies, 26(2), 210-212. https://doi.org/10.7202/007659ar d'utilisation que vous pouvez consulter en ligne.

https://apropos.erudit.org/fr/usagers/politique-dutilisation/ 
Over three decades since founding the Studio, the weavers have experienced an impressive growth technically and artistically. This maturation reached a turning-point in 1978 when the weavers agreed with the aims of consultant Charlotte Lindgren. She established quality standards in design and production, set her sights on the recognition of Inuit tapestries as art (something non-Inuit tapestry weavers have aimed for, alas unsuccessfully ${ }^{5}$ ), and developed a marketing system that is still in place. Between the 1980s and the 1990s the Studio averaged eight exhibitions a year and production could hardly keep up with the demand. The selection for any annual collection, as with the many levels of production, results from the collaborative efforts of the weavers, studio manager, and arts advisor.

I have a few minor criticisms of this outstanding book. Photographs of the weavers at work on the low-warp and high-warp looms would help readers learn about the skills and vision required and the problems presented. When a tapestry is named in the essays, a page number is needed to find the illustration referred to. A map would assist to visualize the vast territories of Nunavik (Arctic Quebec) and Nunavut that the Inuit call home.

As I reflected on this significant body of work I realized that we have before us a record of a luminous legacy, both of the past and the present, of importance to artists especially those who are tapestry weavers, collectors, museologists, historians. A fine publication, Nuvisavik celebrates the high esteem Pangnirtung weavers have won throughout the world by their vibrant tapestries. Through their collective voice they have added to the rich patrimony of their ancestors a new dimension of artistic endeavour.

Betty Kobayashi Issenman

Research Associate

McCord Museum of Canadian History

Montréal, Québec, Canada

\section{WEINSTEIN, Charles \\ Arctique Extrême. Les Tchouktches du détroit de Béring, Éditions Autrement, Collection Monde ${ }^{\circ} 117$.}

Avec l'avancement du progrès techno-scientifique et les politiques d'intégration socio-raciales, les peuples autochtones de la Russie risquent de disparaître. Cela implique la perte d'identités culturelles comme le patrimoine linguistique, les rites et les coutumes qui se sont transmis pendant des siècles et ont été les bases structurelles de la société.

5 Exceptions to this observation are known. Raphael, in the sixteenth century, produced the cartoons for the tapestries of the Sistine Chapel, considered sublime. In the nineteenth and twentieth centuries William Morris, Frank Stella, and David Hockney have had their works translated into tapestries, now deemed to be of high artistic and monetary worth. 
À cause de la colonisation russe, les Tchouktches du détroit de Béring sont donc en train de perdre lentement, mais graduellement, leur identité. Les causes principales - mais non les seules - de cette perte culturelle, sont l'alcoolisme et l'imposition de la langue russe à l'école et dans tous les lieux publics.

Ce phénomène de «russification» également présent dans d'autres réalités ethniques de l'ex-URSS a bien été observé par Charles Weinstein pendant cinq années (1993-1998) de séjour dans la population locale. C'est le sujet de son livre Arctique Extrême.

L'auteur est agrégé de russe et s'intéresse à la littérature des petites ethnies du Grand Nord et de l'Extrême Orient russe. Il a traduit poésies, contes, mythes et à la fin de son expérience, en commentant la conduite d'un compatriote connu à Anadyr, il dit de lui-même: «Je ne suis pas un aventurier en visite chez des sauvages. Je me contente d'étudier la langue des autochtones et de traduire nouvelles, récits, contes et autres matériaux. Certes, j'aimerais plus tard faire connaître cette langue et publier mes traductions, car ce peuple mérite d'être connu.» Selon Weinstein, la littérature et la tradition orale des Tchouktches sont le produit de l'esprit d'hommes en lutte avec un environnement dangereux auquel ils ont su remarquablement s'adapter, comme chasseurs de mammifères marins et éleveurs de rennes.

Le livre, écrit sous forme de journal, est le compte rendu du séjour et des rencontres de l'auteur parmi ce peuple. Avec lui il a partagé la façon de vivre, de penser, de chasser; il s'est conduit comme un des leurs en parlant leur langue et en respectant leurs traditions. Malheureusement, il a aussi constaté l'état d'abandon et de misère, autant spirituel que matériel, dans lequel se trouve la population et les problèmes qu'elle doit affronter qui sont en train de détruire les communautés.

Le problème de l'alcoolisme, commun à beaucoup d'autres ethnies arctiques, est sérieux et risque de devenir un facteur négatif prépondérant à l'intérieur des communautés. À entendre les intéressés, les causes de ce fléau sont à la fois sociales et psychologiques: «les difficultés de la vie quotidienne, le marasme économique, le délabrement de la culture traditionnelle et des langues locales. La population a le sentiment d'être abandonnée, rejetée, méprisée.» À ces difficultés s'ajoutent les problèmes dérivant de la «confrontation avec un autre mode de pensée, une autre langue, une autre éducation. À l'école, on enseigne aux enfants une histoire qui n'est pas la leur. Dans certains villages d'Alaska il n'y a pas de groupe folklorique. Les missionnaires ont lutté contre la culture traditionnelle.»

$\mathrm{Au}$ cours des dernières années beaucoup de choses ont changées - autant politiquement que socialement - et la communauté autochtone ne s'est pas trouvée préparée à affronter des problèmes et des situations inattendus. L'État central russe n'a pas apporté l'aide nécessaire et a contribué à augmenter le déclin social avec des initiatives peu populaires comme l'obligation de payer les médicaments et l'attribution de salaires plus bas aux femmes tchouktches par rapport aux blancs. En ignorant les traditions locales, il a engendré pauvreté et malaise. 
La vie à ces extrêmes latitudes a toujours été difficile mais les populations autochtones ont toujours survécu aux difficultés climatiques et environnementales en respectant la Nature - qu'elles considèrent «une donnée de la vie» - sans transgresser les règles non écrites qui gouvernent le monde arctique. Maintenant cet équilibre s'est brisé - non par leur faute - et risque de ne plus se recomposer. Comme le confirment amèrement les Tchouktches, «l'assimilation des petites ethnies est inéluctable,» avec la conséquence inévitable de la perte inestimable d'un patrimoine humain.

Cesare Censi

Civitanova, Marche, Italie 